\title{
Research on the Improvement of Sea Target Defog Based on Dark Channel Prior
}

\author{
Likun Liu ${ }^{1, a}$, Pengtao $\mathrm{Ni}^{1, \mathrm{~b}}$ \\ ${ }^{1}$ PLA 91550 Unit 94, Liaoning Dalian, 116023, China \\ aemail: tianyafc@163.com, bemail:daliannpt@qq.com
}

Keywords: Dark Channel Prior; Image Enhancement; Image Fusion

\begin{abstract}
There are several error estimates to atmospheric light in the algorithm of image defog based on dark channel prior which the operation efficiency is low. In this paper we propose an improved method to solve these problems. Comparing with the original defogging image, the computing time of our algorithm was shortened by the experimental results. When the sea fog is dense, we adopt image fusion algorithm based on weighted average. This algorithm can improve the color distortion of the restored image, and accurately restore the real scene of the image.
\end{abstract}

\section{Introduction}

The sea fog environment shooting out of the scene visibility and contrast sharply reduced, many features of the image contained will be covered or fuzzy. Thus making the video image surface ship visual system seriously degraded, so the image visual system of ship under the clear sea fog algorithm has high practical significance. In 2009, a method is proposed by He Kaiming based on dark channel prior to the image defog[1], which can effectively achieve a good effect defogging only according to the concentration of the local color of the image to repair the various parts of the color. The atmosphere light will be wrongly estimated when the luminance of target in the scene is similar to the atmospheric light, and optimizing the transmission estimation, through solving the large sparse linear equations, the operation efficiency is low. The memory requirement is very high, can not handle large image resolution. The research goal of this paper is to improve the algorithm based on He Kaiming's theory, and make it more widely used.

\section{Image defog algorithm based on dark channel prior}

\section{Dark channel prior principle}

Firstly, the model of the dark channel prior and the fog image formation is studied, and then the image de fog based on the improved dark channel theory is carried out.

$$
\operatorname{Dark}(x)=\min _{c \in\{r, g, b\}}\left(\min _{y \in \Omega(\mathrm{x})}\left(J^{c}(\mathrm{y})\right)\right.
$$

Which $J^{c}$ is a channel 3 channel of the image, $\Omega(x)$ is a window to $x$ pixel as the center,

$\operatorname{Dark}(x)$ said the dark channel corresponds to the gray value of $x$ in the point of the original images at. Significance of formula (1):First, find the minimum value of each pixel in the RGB component, in a pair of the same size of the original image and gray image, and then the gray scale images of 
minimum filter, filter radius is determined by the window size, generally Windows Size $=2 *$ Radius +1 . Dark channel prior theory:

$$
J^{\text {dark }} \rightarrow 0
$$

This theory is defined as dark channel a priori. After a large number of similar images of statistics, it is found that the gray black channel clear image corresponding to the sunny weather conditions for about $75 \%$ of the pixel value is 0 , the pixel intensity of the dark channel also has about $90 \%$ of the value of less than 25 .

\section{Estimation and optimization of transmittance}

$$
I(x)=J(x) t(x)+A(1-t(x))
$$

Among them, $I(x)$ (fog image), $J(x)$ is to restore the fog image, $A$ the global atmospheric light components, $t(x)$ as the transmittance. Now the known condition is $I(x)$ that $J(x)$ is the target value, obviously, this is a solution of the equation, and therefore need a priori. By formula (3):

$$
\frac{I^{c}(x)}{A^{c}}=\frac{J^{c}(x)}{A^{c}} t(x)+1-t(x)
$$

As mentioned above, the superscript $\mathrm{C}$ indicates the meaning of the RGB three channels. The first assumption is that the transmittance in each window is constant, defined as it is, and the value has been given, then the formula (4) on both sides of the two minimum value calculation, get the next type:

$$
\min _{y \in \Omega(\mathrm{x})}\left(\min _{c} \frac{I^{c}(y)}{A^{c}}\right)=\tilde{t}(x) \min _{y \in \Omega(\mathrm{x})}\left(\min _{c} \frac{J^{c}(y)}{A^{c}}\right)+1-\tilde{t}(x)
$$

In the formula, is to seek the fog image, according to the dark channel prior theory:

$$
\operatorname{Dark}(x)=\min _{y \in \Omega(x)}\left(\min _{c \in \Omega(r, g, b)} J^{c}(y)\right)=0
$$

Therefore,

$$
\min _{y \in \Omega(\mathrm{x})}\left(\min _{c} \frac{J^{c}(y)}{A^{c}}\right)=0
$$

Plugged (7) to (5),

$$
\tilde{t}(x)=1-\min _{y \in \Omega(x)}\left(\min _{c} \frac{I^{c}(y)}{A^{c}}\right)
$$

$\tilde{t}(x)$ is an estimated value of transmittance. In fact, even if it is sunny clouds, also exist in the air with some particles, therefore, it is necessary to retain a certain degree of fog in time to fog, which can be adopted in the formula (8) in the introduction of a [0,1] factor, then type (8) for correction

$$
\tilde{t}(x)=1-\omega \min _{y \in \Omega(x)}\left(\min _{c} \frac{I^{c}(y)}{A^{c}}\right)
$$


The transmittance of dark channel prior to obtain only a rough estimate, the transmittance characteristics description of fog image is not accurate.Therefore, He use the meothod of Soft Matting to optimize the transmittance[2]. As is shown in Figure 1.

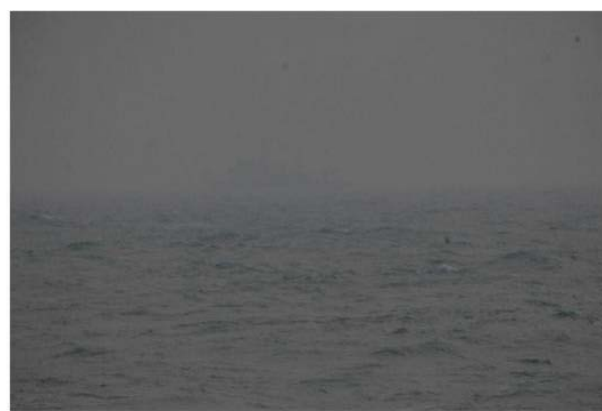

Figure 1-a Foggy image

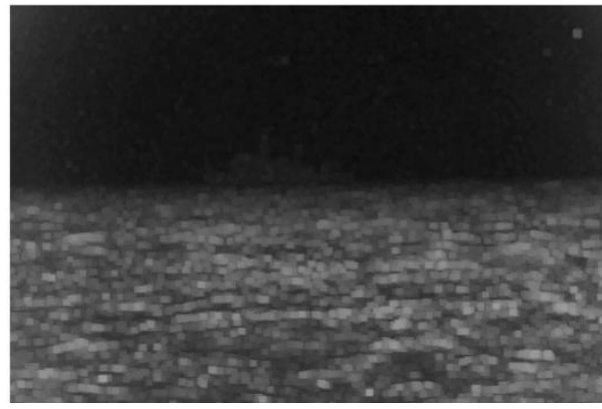

Figure 1-c Estimation of transmittance

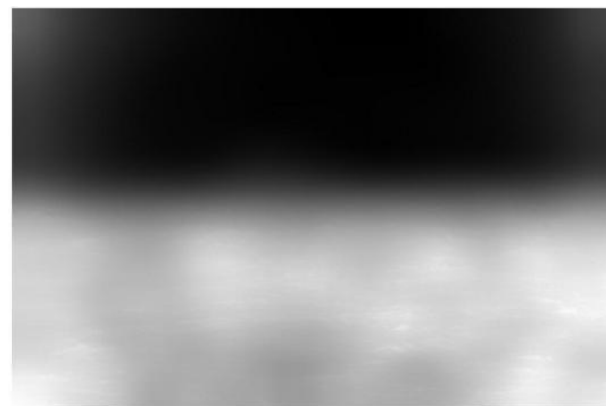

Figure 1-e Taransmission optimization

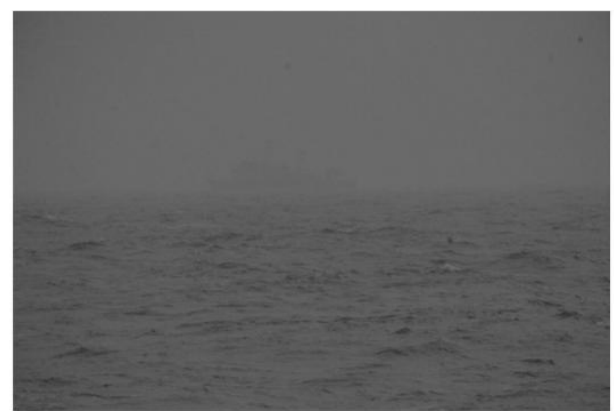

Figure 1-b Minimum filtering

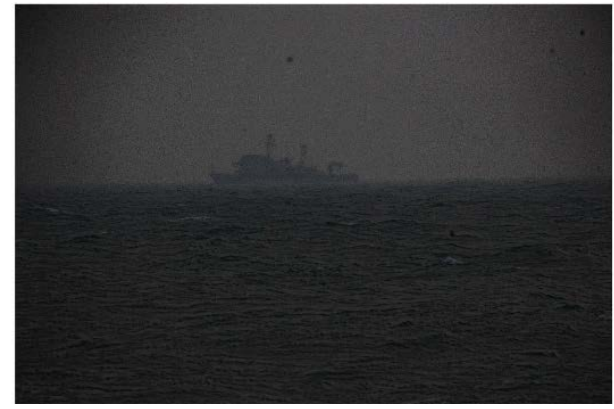

Figure 1-d Preliminary recovery

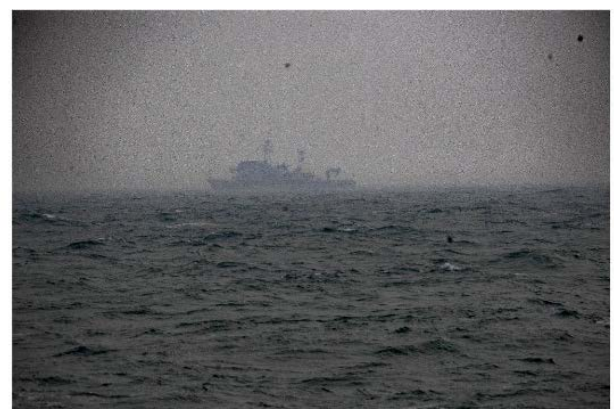

Figure 1-f Recovery image

Figure 1 The defog method of $\mathrm{He}$

\section{Atmospheric light estimation}

Estimation of atmospheric light A researchers have proposed different methods in estimation of atmospheric light, Tan[3] proposed directly pixel fog image in the highest brightness value of the pixel as the sky light, this estimation method is actually not accurate. Because in the fog image if there is white or high brightness objects will affect the estimated value.

When the concentration of the fog is larger, the value of the dark channel is larger. So the fog image of the dark channel in a certain extent can reflect the distribution of fog, Thus the atmospheric light estimation can be carried out by the dark passage of the fog image. He proposed to estimate the sky light method is to first according to the image of the dark channel, the dark channel pixels in accordance with the size of the size of the order, and then take the top $0.2 \%$ pixels in these pixels. The location of these pixels is representative of the larger fog concentration in the actual scene. Then take the highest gray value in all the pixels as the sky light A. 


\section{Fog image restoration}

After obtaining the transmission rate and atmospheric light, the final clear image can be calculated by the atmospheric scattering model (2-15), and its mathematical expression is as follows:

$$
J(x)=\frac{I(x)-A}{t(x)}+A
$$

Because the transmission rate tends to 0 .So in the calculation of the restored image part of the sky when will be very sensitive to noise.In order to avoid this situation, it will set a lower limit for the $t$, thus enhancing the robustness. Then the calculation formula of atmospheric scattering model based on fog reduction:

$$
J(x)=\frac{I(x)-A}{\max \left(t(x), t_{0}\right)}+A
$$

\section{Algorithm improvement}

The original algorithm is fast enough, there are too many floating-point calculations, although you can try to optimize the deep level of code, including SSE processing, parallel operation, but due to the implementation of the algorithm is sequential, not full parallel, when consumption increases. Direct to the fog after the image will be more than the original dark, so the need for a certain exposure after the treatment is enhanced, but the general algorithm is often not considered enhanced. In view of the above mentioned to the fog algorithm operation number and fog after the visual effect of the two aspects, this paper puts forward two aspects of improvement measures.

1, Studies have shown that [4] algorithm, since the dark channel transmittance than to fog the rest to fine, if appropriate a little lower precision, the defogging effect theory should not be too much difference. Based on the calculated transmittance, and not the original strike, but the first of the original samples, calculate the transmittance of small map, and then get the original image by transmittance about interpolation method.

2, The algorithm to image to fog fog in the preliminary treatment, and contrast stretching, Gamma correction, histogram equalization method for image enhancement, then the weighted average image fusion to get the final image to the fog.

First we need to obtain the weights of the initial fog image $J_{1}$ and the contrast improved image

$J_{2}$, which is $m_{1}$ and $m_{2}$. When the reference image are obtained by subtraction method is simple to improve the contrast of the original image and the original fog, fog image in the foreground part compared to the vision part of gray value is low, after subtraction of the whole image, close part will appear the loss of information. So in the last part of the fusion image in close proportion to low. The transmission rate is inversely proportional to the depth of field, transform form transmission rate as weight map. Since a better effect in definition, we increase the proportion of the initial de fog image. So,

$$
\begin{aligned}
& m_{1}(x)=\min ((k+t(x)), 1) \\
& m_{2}(x)=1-m_{1}(x)
\end{aligned}
$$


According to the weighted average image fusion algorithm,

$$
F(x)=m_{1}(x) J_{1}(x)+m_{2}(x) J_{2}(x)
$$

We get the initial fog image and the contrast improved image to fuse. Which $m_{1}$ and $m_{2}$ corresponds to the weight of $J_{1}$ and $J_{2}$, and. $m_{1}+m_{2}=1$.

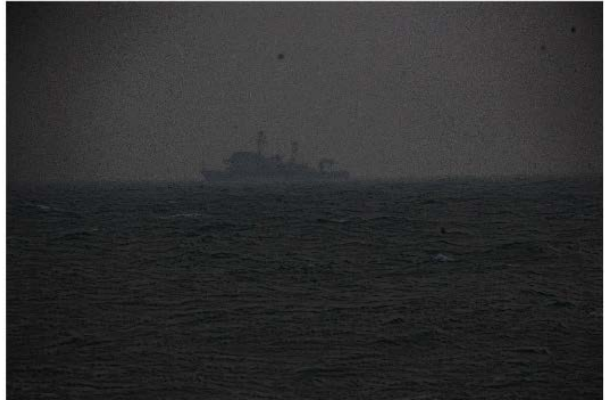

Figure 2-a Primary restoration image

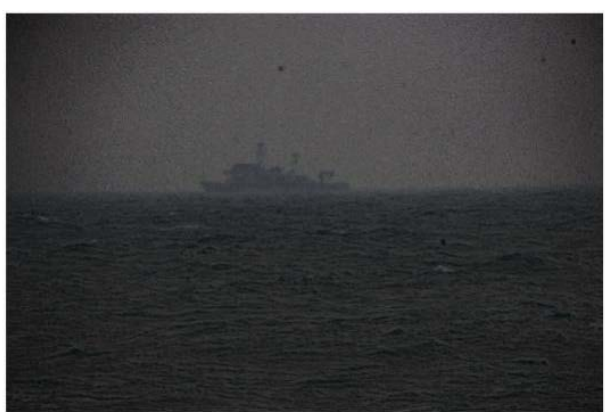

Figure 2-c Primary restoration with $\mathrm{m} 1$

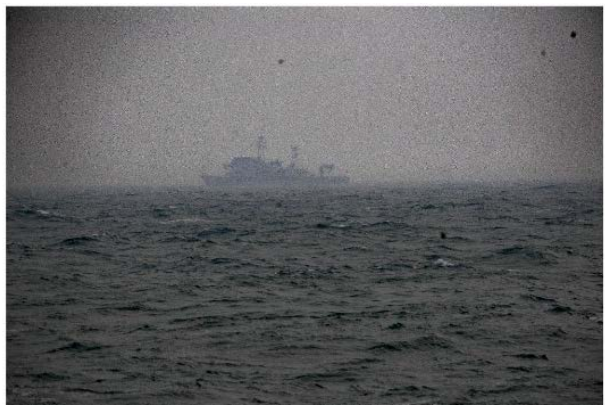

Figure 2-e Taransmission optimization

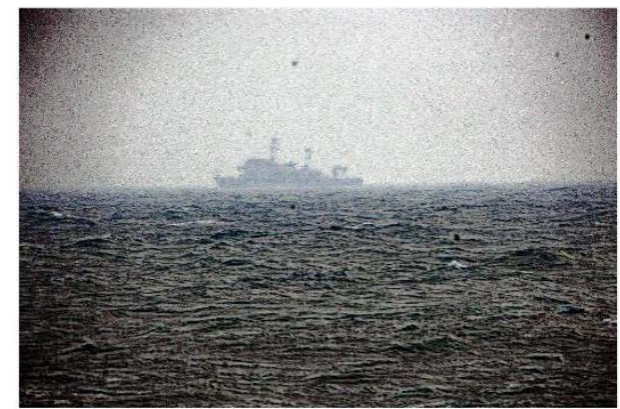

Figure 2-b Histogram equalization image

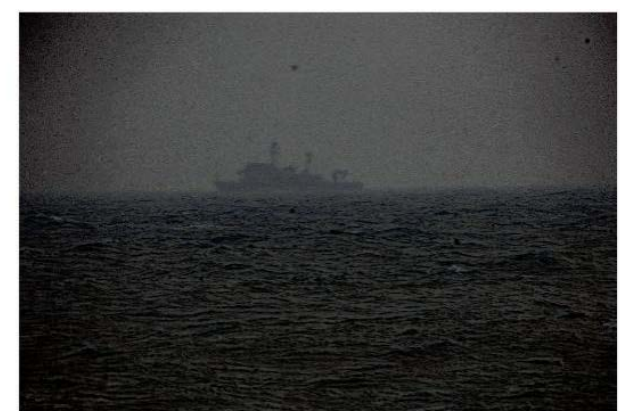

Figure 3-d Histogram equalization with $\mathrm{m} 2$

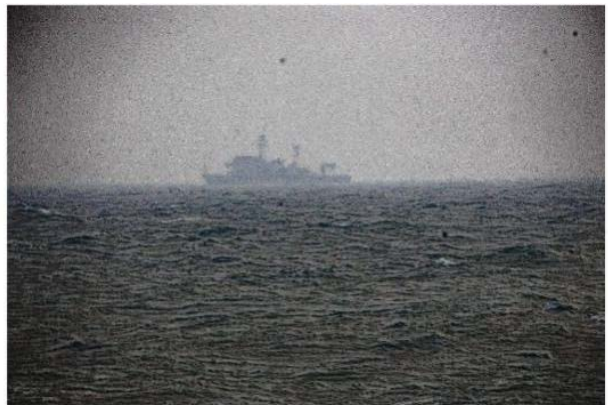

图 2-f Improved algorithm in this paper

Figure 2 The defog method of this paper

\section{Applications and results}

For floating-point computation of the original algorithm, slow speed characteristics, by sampling the original image, the size of the original resolution into the calculated transmittance small map, and then get the original image by transmittance about interpolation method.

The use of MatlabR2014a in CPU Core (TM) i7-4790, 16G of physical memory, a 64 bit Win7 operating system, for a $6048 * 4032$ color picture, the original algorithm needs 25.456 s. For the same color image based on the improved sampling method to the fog drop time to $21.274 \mathrm{~s}$, saving time $4.182 \mathrm{~s}$.

The final result of the improvement of the visual effect after the fog is shown in figure 2. From 
the results of the image can be seen after preliminary restoration, image color distortion and brightness of dark, black color. The weighted average color correction based on image fusion, image rendering results color is more real and original preliminary reconstruction of fog image (Figure 2-A) compared to clarity has improved greatly; and the original algorithm optimized transmittance recovery plan (Figure 2-e) comparison, visibility is also improved.

\section{Conclusion}

In order to fog image processing, to improve the clarity of the image, the first analysis of the dark channel prior theory and image formation model, then the prior theory was improved, the results showed that: compared with the He image defogging algorithm, this algorithm reduced the time near the sea fog; relatively strong, the the weighted average image fusion algorithm based on this method can better improve the color image of the distortion problem, accurate reduction of the real scene image.

\section{Reference}

[1] He K M, Sun J, Tang X O. Single image haze removal using dark channel prior[C]. In: Proceeding of IEEE Conference on Computer Vision and attern Recognition. Washington D.C, USA: IEEE, 2009:1956-1963

[2] He K M, Sun J, Tang X O. Guided Image Filtering. Proceedings of the $11^{\text {th }}$ European Conference on Computer Vision, 2010.

[3]Tan R T. Visibility in bad weather from a single image[C]. Proceedings of IEEE Conference on Computer Vision and Pattern Recognition, 2008, 1-8.

[4] Zhang $\mathrm{H} \mathrm{Y}$, Zhang S N, Wu Y D. Fast defogging method based on human visual propertied[J].Computer Application, 2014,34(06): 1753-1757,1761. 